The proof is completed by observing that

$$
\int_{0}^{1} \Omega(k f) d \mu \geqq \sum \Phi\left(2 c_{m_{n}}\right) \mu\left(E_{m_{n}}\right) \geqq \sum_{n=1}^{\infty} \alpha s_{0} .
$$

REFERENCE

1. Krasnosel'skii and Rutickii, Convex functions and Orlicz spaces, P. Noordhoff Ltd., Groningen, 1961.

NORTHWESTERN UNIVERSITY

\title{
ON A COMBINATORIAL PROBLEM OF ERDÖS
}

\section{KLEITMAN}

Let $C(n, m)$ denote the binomial coefficient $n ! /(m ! n-m !)$. Let $S$ be a set containing $N$ elements and let $X$ be a collection of subsets of $S$ with the property that if $A, B$ and $C$ are distinct elements of $X$, then $A \cup B \neq C$. Erdös [1], [2], has conjectured that $X$ contains at most $K C(N,[N / 2])$ elements where $K$ is a constant independent of $X$ and $N$. The problem is related to a result of Sperner [3] to the effect that if the collection $X$ has the more restrictive property that no element of $X$ contains any other, then $X$ can have at most $C(N,[N / 2])$ elements.

We show below that Erdös' conjecture for $K=2^{3 / 2}$ can be deduced directly from Sperner's result.

Let $L_{N}$ be defined by

$$
\begin{aligned}
L_{N} \equiv & 2^{[N / 2]} C\left(N-[N / 2],\left[\frac{1}{2}(N-[N / 2])\right]\right) \\
& +2^{N-[N / 2]} C([N / 2],[N / 4]) .
\end{aligned}
$$

An easy calculation shows that $L_{N}$ is always less than $2^{3 / 2} C(N,[N / 2])$ to which it is asymptotic for large $N$. We prove:

THEOREM. If $X$ is a family of subsets of an $N$ element set $S$ such that no three distinct $A, B, C$ in $X$ satisfy $A \cup B=C$, then $X$ has less than $L_{N}$ elements.

Proof. For any finite set $T$ and family $X$ of subsets of $T$ define

$$
m_{T}(X) \equiv\{A \in X \mid B \in X \text { and } B \subset A \text { imply } B=A\} .
$$

Received by the editors March 9, 1965. 
Note that $m_{T}(X)$ satisfies the hypothesis of Sperner's theorem and hence $m_{T}(X)$ contains at most $C(M,[M / 2])$ where $M$ is the number of elements in $T$.

Let $S=T_{1} \cup T_{2}$ where $T_{1} \cap T_{2}=\varnothing$ and $T_{1}$ contains [N/2] elements. For each subset $A \subset S$ let

$$
D_{j}(A)=\left\{B \in X \mid B \cap T_{j}=A \cap T_{j}\right\}, \quad j=1,2 .
$$

Note that $m_{s}\left(D_{2}(A)\right)$ and $m_{T_{1}}\left(\left\{B \cap T_{1} \mid B \in D_{2}(A)\right\}\right)$ have the same number of elements. In consequence, since $T_{1}$ has $[N / 2]$ elements, $m_{s}\left(D_{2}(A)\right)$ can have at most $C([N / 2],[N / 4])$ elements. Similarly $m_{s}\left(D_{1}(A)\right)$ can have at most $C\left(N-[N / 2],\left[\frac{1}{2}(N-[N / 2])\right]\right)$ elements.

Next we show that if $A \in X$ then $A \in m_{s}\left(D_{1}(A)\right) \cup m_{s}\left(D_{2}(A)\right)$. Suppose $A \in X$ and $A \notin m_{s}\left(D_{1}(A)\right) \cup m_{s}\left(D_{2}(A)\right)$. Then there are subsets $B_{1}$ and $B_{2}$ such that $B_{j} \cap T_{j}=A \cap T_{j}, B_{j} \neq A, B_{j} \subset A, B_{j} \in X, j=1,2$. But then, $B_{1} \cup B_{2}=A$ and $B_{1}$ and $B_{2}$ and $A$ are distinct and hence $A \in X$. Thus we have shown that $X \subset \bigcup_{A \in X}\left\{\left(m\left(D_{1}(A)\right) \cup m\left(D_{2}(A)\right)\right\}\right.$.

Note that $m_{s}\left(D_{1}(A)\right)=m_{s}\left(D_{1}(B)\right)$ if $A \cap T_{1}=B \cap T_{1}$. Hence there are at most $2^{[N / 2]}$ distinct families $m_{\mathbf{s}}\left(D_{1}(A)\right)$, one for each distinct $A \cap T_{1}$. Similarly, there are at most $2^{N-[N / 2]}$ distinct families $m_{s}\left(D_{2}(A)\right)$. Hence the number of elements in $X$ is at most $L_{N} . L_{N}$ can be reduced by $C([N / 2],[N / 4]) \cdot C\left(N-[N / 2],\left[\frac{1}{2}(N-[N / 2])\right]\right)$ by taking into account the overlap between the elements of the

$$
m_{s}\left(D_{1}\right) \text { 's and } m_{s}\left(D_{2}\right) \text { 's. }
$$

The proof above makes use of only part of the hypothesis; namely, that $X$ contains no subset $A$ which is a union of two others, $B$ and $C$, with

$$
\begin{aligned}
B \cap T_{1} & =A \cap T_{1}, \\
C \cap\left(S-T_{1}\right) & =A \cap\left(S-T_{1}\right),
\end{aligned}
$$

for a given [N/2] element subset $T_{1}$ of $S$. One can construct an $X$ satisfying these conditions with only $2 C([N / 2],[N / 4]) C(N-[N / 2]$, $\left.\frac{1}{2}[N-[N / 2]]\right)-1$ elements fewer than the maximum noted above, so that $2^{3 / 2} C(N,[N / 2])(1+o(N))$ is a best bound, for families $X$ subject to this weaker restriction.

The upper limit $2^{3 / 2}$ deduced for $K$ above is not a best estimate under the more general limitation on $X$ suggested by Erdös. If we use the fact that the intersections with $T_{1}$ of the elements of the $D_{2}$ 's must form a family satisfying our hypotheses for the $[N / 2]$ element set $T_{1}$, the estimate for $K$ given above can be reduced by approximately 5 percent for large $N$. The best value for $K$ is probably 2 
(realized for $N=1$ ) and, if the maximum number of elements of $X$ is written as $K_{N} C(N,[N / 2])$ it may be that $K_{N}$ approaches as $N$ increases.

The result may be straightforwardly extended to collections $X$ restricted such that no element contains the union of $j$ others. One can deduce $j^{3 / 2} C(N,[N / 2])$ as upper limit on the number of elements in such an $X$.

I would like to thank Dr. E. Brown for his help.

\section{BIBLIOGRAPHY}

1. P. Erdös, On a lemma of Littlewood and Offord, Bull. Amer. Math. Soc. 51 (1945), 898-902.

2. S. Ulam, A collection of mathematical problems, Interscience, New York, 1960, p. 27.

3. E. Sperner, Ein Satz ibber Untermengen einer endlichen Menge, Math. Z. 27 (1928), 544-548.

BRANDEIS UNIVERSITY 